\title{
Measurement Standards for Low and Medium Peak Pulse Voltages
}

\author{
A. R. Ondrejka* and P. A. Hudson* \\ National Bureau of Standards, Boulder, Colo.
}

(November 10, 1965)

\begin{abstract}
Two systems for the measurement and standardization of peak pulse voltage have recently been developed in the Radio Standards Engineering Division of the National Bureau of Standards. Designed for use with pulses having durations as short as 10 nanoseconds, the systems represent the initial effort by NBS for establishment of standards in this field and it is expected that they will serve as the basis for a calibration service.

One of the systems is a refined, slideback voltmeter employing a solid state diode. The rise time is less than 10 nanoseconds and the instrument is useful for measurements in the range $5 \mathrm{~V}$ to $100 \mathrm{~V}$ as presently constructed.

The second system standardizes the output peak voltage level of pulse generators by means of cascaded zener diodes. The clipping level of the diodes is determined first by d-c voltage measurements and the system is subsequently used to generate pulses of known peak amplitudes. The rise time of the diodes is approximately 30 nanoseconds.

The accuracy of both systems is within 0.25 . percent, and comparison measurements have been made between the two, and with other NBS a-c and $\mathrm{rf}(\mathrm{CW})$ voltage standards.
\end{abstract}

Key Words: Measurement, peak, pulse, pulse-limiting, slideback, standard, voltage.

\section{Introduction}

The use of pulse waveforms has increased significantly during recent years, resulting in a wider interest in measurement of pulse parameters. Pulses are used in a variety of applications such as high speed electronic computers, medical applications, pulse amplitude modulation of carrier waves, and for testing of broadband circuits and components. The time domain reflectometer is a good example of the latter. In addition, progress has been made in the state of the art of generation of fast-rise, short-duration pulses. Subnanosecond rise times are now common and work is progressing on picosecond rise-time pulsers.

The parameters of interest in measurements on pulses include the rise time and the peak voltage amplitude. Various types of instruments for performing these measurements are commercially available, the most common of which is the cathode ray oscilloscope. Other types include direct reading peak voltmeters which cover a wide dynamic range from a few millivolts to several kilovolts at pulse widths down to a fraction of a microsecond. The specified uncertainties of the instruments used for peak voltage measurement are generally 1 to 2 percent.

As a result of the wider application of pulse waveforms and the overall higher accuracies required in present-day measurements, the National Bureau of Standards has received requests for calibration services in the pulse parameters. It is the purpose of this paper to describe two systems for measurement and standardization of peak pulse voltage in the range $5 \mathrm{~V}$ to $100 \mathrm{~V}$ for pulses having durations of at least 10 nsec. These systems represent the initial efforts at NBS for establishing standards in this field and it is expected that they will serve as the basis for a calibration service.

Two independent systems were developed to allow intercomparison and thus better evaluate the absolute accuracies. One system is designed to measure the pulse voltage across its input terminals while the other generates a predetermined, accurately known, pulse voltage level. Intercomparison can then be carried out without the need for a transfer instrument.

The development of standards for pulse rise time measurement is planned for the future.

\section{Principles of Operation and Design}

The voltage waveform of interest here is a train of so-called "d-c" or unidirectional pulses [1]. ${ }^{1}$ That is, ideally (in the mathematical sense), the voltage excursion occurs in only one direction from the zero position. Usually the pulse width is much less than the time interval between pulses and the average voltage is small compared to the peak voltage. One of the problems is to obtain an accurate, continuous reading of a waveform whose instantaneous value is zero the greater part of the time. 


\subsection{The Standard Measurement System}

In the design of a system, for peak pulse voltage measurement, there are certain features which are necessary or desirable. Of primary importance is the system accuracy which requires that the design eliminate as many sources of error as possible even though some sacrifice in ease of operation may have to be made. Other desirable features include wide dynamic range, insensitivity to pulse duty cycle, and a continuous output reading. Since it is desired to use the system for measurements on fast-rise, shortduration pulses, it must also have an extremely broad bandwidth.

After theoretical and experimental investigation of various known measurement techniques, it was decided that a system based on the slideback principle would adequately fulfill the requirements mentioned above. Slideback voltmeters have been in use for a number of years and have been described in the literature $[2,3]$. The principle involves comparing the unknown voltage to an accurately known, adjustable voltage. The circuit is arranged so that the two voltages oppose each other and null detection methods are used to indicate when the voltages are equal. For the measurement of d-c voltage, high accuracy and precision are attainable. In applying the principle to pulse voltage measurement a diode is employed to limit current drain from the d-c slideback source during the time interval between pulses. This time interval may be several orders of magnitude (e.g., 104) greater than the pulse width. The version used here can be described by referring to the circuit in figure 1 , which is included for the purpose of illustrating the principle. In the absence of an input signal and with the d-c slideback voltage set to zero, no current flows in the circuit as evidenced by a null indication on the galvanometer. When a train of pulses (positive pulses for the circuit shown) is applied to the input, the diode becomes conducting and may be returned to its original nonconducting state by adjusting the reverse d-c (slideback) voltage until the diode is cut off and the galvanometer is again nulled. The reverse $\mathrm{d}$-c bias voltage, which can be easily and accurately measured, is then equated to the peak pulse voltage.

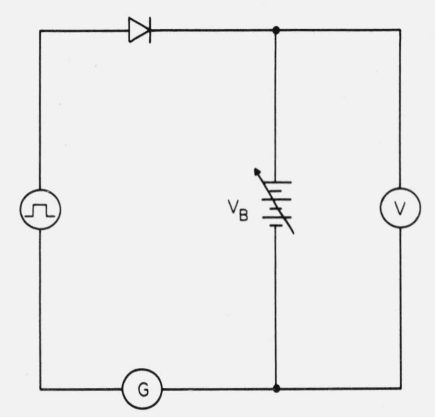

FIGURE 1. Basic circuit of slideback principle.
The accuracy of the system is primarily dependent on the validity of the assumption that, at a null condition, the d-c bias voltage is equal to the peak pulse voltage. The validity of this assumption is, in turn, a function of the characteristics of the diode. In an ideal diode both the back resistance and the forward conductance would be infinite while the rise time and shunt capacitance would be zero. The problem then was to choose an available diode having as nearly as possible these ideal characteristics. One of the critical characteristics is the forward conductance at low values of applied voltage. The forward conductance is important because of the finite sensitivity of the null detector. If some voltage, $V_{e}$, across the diode is required to cause a detectable current to flow, then the d-c slideback voltage, $V_{B}$, would be less than the peak pulse voltage, $V_{p}$, by an amount $V_{e}$ which we have called the offset voltage. It is possible to measure the $V_{e}$ of a particular diode and apply a correction. Some uncertainty would, however, be involved in this correction and, in general, it is desired to keep the correction as small as possible.

The forward diode current, $I$, is given approximately by the equation [4]

$$
I=-I_{s}\left(\epsilon^{q v / k T}-1\right)
$$

where

$$
\begin{aligned}
I_{s} & =\text { reverse saturation current } \\
q & =\text { electronic charge } \\
v & =\text { applied voltage } \\
k & =\text { Boltzmann's constant } \\
T & =\text { Temperature, }{ }^{\circ} \mathrm{K} .
\end{aligned}
$$

Thus the forward current is a function of the reverse current, $I_{s}$. This suggests the choice of certain types of point-contact diodes which have relatively large reverse currents. In the point-contact diodes tested, the reverse current did not reach a saturation value but, rather, tended to increase with increasing reverse voltage. The resultant heating effect caused changes in the forward characteristics. Thus the pointcontact types were not suitable for this application. Junction diodes, on the other hand, are usually characterized by extremely low reverse currents and would not be suited because of the resulting large forward offset voltage. A diode type which is intermediate between these two is the metal-semiconductor type. In one version of this type, a gold wire is welded to $n$-type germanium to form a rectifying contact as in the $1 N 270$ diode. The reverse resistance of the 1 N270 is approximately $10 \mathrm{M} \Omega$ as compared to $1000 \mathrm{M} \Omega$ for typical junction types. A comparison of $V-I$ curves for the $1 \mathrm{~N} 270$ and a $1 \mathrm{~N} 4445$ junction diode is shown in figure 2 . The conductance of the $1 \mathrm{~N} 270$ at the voltage levels shown is approximately 600 times greater than that of the junction type. Since conduction in metalsemiconductor diodes is by majority carriers, they have a better high frequency response (fast rise time) and in addition the shunt capacity is comparatively small. The rise time of the $1 \mathrm{~N} 270$ is approximately $10 \mathrm{nsec}$ and at present it appears to have the best overall 


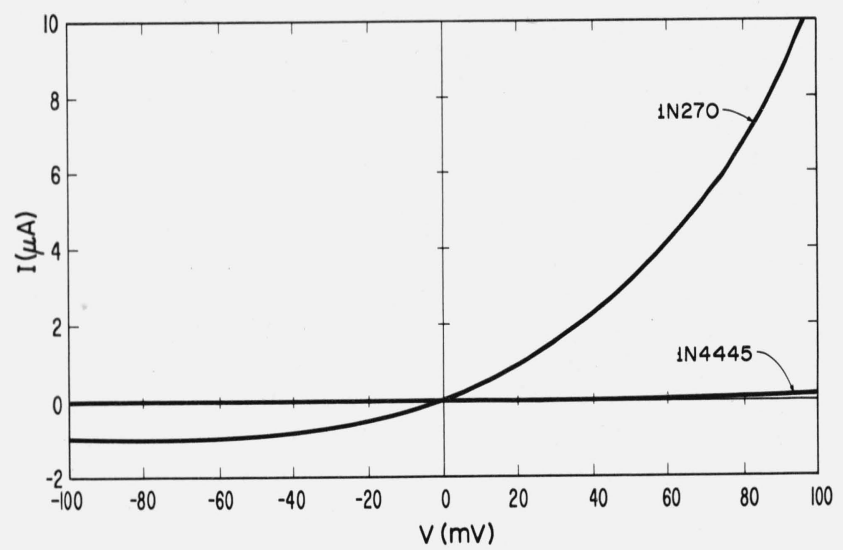

FIGURE 2. Comparison of V-I characteristics of 1 N270 gold bonded diode and 1 N4445 junction diode.

characteristics of the several diodes tested for this application.

Another important factor in the accuracy of the system is the means used for detecting when the diode is just at cutoff. The galvanometer detector mentioned earlier is not suitable because it responds to average current and thus suffers a considerable loss in sensitivity over that obtainable with a peak-responding detector. In addition, nulling the galvanometer would not guarantee equality between the pulse and d-c slideback voltages because of the reverse leakage current through the diode during the time interval between pulses. This current tends to reach a saturation value, $I_{s}$, of the order of $1 \mu \mathrm{A}$ (see fig. 2). The average reverse current, $\bar{I}_{R}$, then is given by

$$
\overline{I_{R}}=I_{s}\left(1-f t_{p}\right)
$$

where $f$ is the pulse repetition rate and $t_{p}$ is the pulse duration. Typical pulse duty cycles, $f t_{p}$, range from $10^{-4}$ to $10^{-1}$ and thus $\overline{I_{R}}$ is equal to at least $0.9 I_{s}$. The average forward current through the diode is given by

$$
\overline{I_{F}}=I_{p} f t_{p}
$$

where $I_{p}$ is the peak current and a flat-top pulse is assumed. At null, $\overline{I_{F}}=\overline{I_{R}}$, and

$$
I_{p}=\frac{I_{s}\left(1-f t_{p}\right)}{f t_{p}} .
$$

When the pulse duty cycle is $10^{-1}$, for example, $I_{p}=9 I_{s}$, or approximately $9 \mu \mathrm{A}$. Referring again to figure 2 , this current corresponds to $80 \mathrm{mV}$ forward voltage and the peak pulse voltage must be larger than the $d-c$ bias by this amount in order to null the galvanometer. At lower duty cycles, the pulse voltage would need to exceed the d-c bias by even greater amounts.

Due to the fact that the pulse duty cycle for narrow pulses is difficult to measure accurately and the reverse saturation current, $I_{s}$, varies with temperature, it is not practical to apply a correction for this effect.

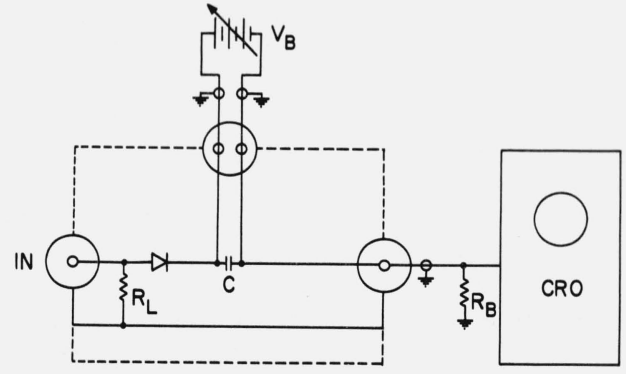

FIGURE 3. Circuit diagram of slideback voltmeter system.

A better detector was devised by substituting a coaxial resistor and a high gain cathode ray oscilloscope for the galvanometer. The circuit diagram of the complete measuring system is shown in figure 3 . This is a peak detecting system and does not depend on average values of d-c current as previously. The CRO is set for capacitive coupling across the resistor, $R_{B}$. Pulses of current flowing through $R_{B}$ are detected by the CRO and the d-c bias voltage is adjusted so that the top of the pulse just recedes into the baseline. The baseline is not affected appreciably by the reverse current ${ }^{2}$ except for a very small differentiated pulse at the leading and trailing edges of the input pulses which is caused by the shunt capacitance of the diode.

Since the CRO detector has limited sensitivity, there will be some small forward pulse current, $I_{e}$, through the diode which is not detectable. As mentioned earlier, the voltage, $V_{e}$, which causes this current to flow is the difference (offset) between the peak pulse voltage and the $\mathrm{d}-\mathrm{c}$ bias voltage. That is, $V_{e}=V_{\text {pulse }}-V_{\text {d-c. }}$. Thus, when the d-c bias voltage has been adjusted to cause the peak of the pulse to just recede into the baseline, the diode will appear to be cutoff when in reality a small current continues to flow. It is possible to estimate the magnitude of the offset voltage by use of the $V \cdot I$ curve of figure 2 and a knowledge of the detector sensitivity. The detector sensitivity is $5 \mathrm{mV} / \mathrm{cm}$ and resolution is approximately $0.2 \mathrm{~mm}$ on the oscilloscope CRT which corresponds to a minimum detectable voltage of $0.1 \mathrm{mV}$. This voltage is the $I R$ drop across the $50 \Omega$ coaxial resistor, $R_{B}$, which terminates the coaxial line to the oscilloscope input and thus the minimum current is $2 \mu \mathrm{A}$. Referring to figure 2 , it can be seen that at this current the offset voltage is $40 \mathrm{mV}$. This voltage is added to the measured $\mathrm{d}$-c bias voltage as a correction. Due to thermal effects the offset voltage may vary $\pm 10 \mathrm{mV}$ and this figure is taken as an uncertainty and it is independent of the input voltage level.

\footnotetext{
${ }^{2}$ It should be noted that when the bias voltage is equal to the input pulse voltage the reverse current goes to zero during the time the pulse is impressed across the diode. This interruption of the reverse current results in the generation of a small positive pulse. Since the reverse current is approximately $1 \mu \mathrm{A}$ and the resistor is $50 \Omega$, the pulse voltage Since the reverse current is approximately $1 \mu \mathrm{A}$ and the resistor is $50 \Omega$, the pulse voltage
is $50 \mu \mathrm{V}$. The top of this pulse is the true baseline; however, due to the limited sensitivity of the CRO it appears only as a widening of the trace.
} 
The detector sensitivity limits the ability of the operator to determine when the diode is just at cutoff and this is another factor which affects the accuracy of the measuring system. It was found experimentally that the random error caused by this factor alone resulted in variations in the reading of the peak pulse voltage as large as $\pm 20 \mathrm{mV}$ from the mean. In order to make a quantitative determination of this error, the standard deviation of the mean was calculated for a sample of 30 measurements made with the input voltage held constant. This value was calculated to be $\pm 10 \mathrm{mV}$ and was taken as an additional error which, again, is independent of the input pulse voltage level.

The other source of error is in the measurement of the d-c bias voltage. This measurement is made with a digital voltmeter which is accurate to within 0.05 percent of the indicated voltage. The overall system accuracy is estimated to be within 0.05 percent $\pm 20 \mathrm{mV}$.

To complete the description of the measuring system, reference is made to figure 3 . That portion of the circuit enclosed by the dashed lines is mounted in a small, round, metal container $1 \frac{1 / 2}{2}$. in diameter and $2 \frac{1 / 2}{}$ in. long. The load resistor, $R_{L}$, consists of three film resistors connected in parallel. These resistors are easily removed so that $R_{L}$ may be adjusted to match any generator impedance from 50 to $10,000 \Omega$. The parallel arrangement reduces the inductive reactance as compared to a single resistor and also increases the power dissipation capability. A second mount employing a $50 \Omega$ coaxial resistor is used for measurements on pulses having the fastest rise times.

The slideback voltage is obtained from a battery pack containing ten $9 \mathrm{~V}$ batteries and one $12 \mathrm{~V}$ battery. Any or all of the batteries may be switched into the circuit and a 10-turn potentiometer across the $12 \mathrm{~V}$ battery serves as a vernier. The capacitor, C, connected across the battery serves as a path for the pulse current. Batteries cause much less distortion of the pulse than the electronically regulated supplies which were tried. The reason for the distortion by the regulated supplies has not been investigated extensively, but it is believed to be due to the filter capacitors and the frequency dependence of the internal impedance. Connection between the diode mount and the bias supply is made by means of a shielded cable. A $50 \Omega$ cable is used to connect to the oscilloscope detector and this cable is terminated by the $50 \Omega$ coaxial load, $R_{B}$, which is connected in parallel with the CRO input. The bandwidth of the CRO vertical amplifier is $50 \mathrm{MHz}$.

Since $R_{L}$ may be as large as $10,000 \Omega$, the slide back system may also be used as a high impedance voltmeter. That is, it can be connected in parallel with the output of a pulse generator to measure and monitor the voltage level. The loading effect due to $R_{L}$ is, in most cases, negligible.

\subsection{The Standard Generating System}

In order to provide a cross-check on the measuring system described above, a second independent system was developed. It was desired that the second system standardize the output level of a pulse generator to an accurately predictable value. In addition it was desirable that the output level be constant over relatively long periods of time. Intercomparison of this system and the first could then be carried out without the need for a transfer standard.

The most promising method for achreving these objectives appeared to be a circuit employing zener (reference) diodes to clip or limit the pulse generator output. The voltage level at which limiting occurred could be determined using accurately measured d-c voltage. It would then be assumed that pulse voltage limiting would occur at the same level. In order to establish the validity of this assumption, an investigation of the characteristics of zener diodes and their associated circuits was required.

The regulating action of zener diodes is now fairly well understood. This action occurs in reverse biased junction diodes when the reverse voltage, $V$, exceeds a certain critical value, $V_{c}$. The diode current increases extremely rapidly with $V>V_{c}$ causing the voltage drop across the junction to maintain a nearly constant value as shown in figure 4 [5]. The rapid increase in current is caused by an avalanche effect in the semiconductor material and is due mainly to majority (i.e., electron) carriers. Thus, the action can be fast and is highly desirable for pulse voltage limiting to avoid undue increase in the rise time of the output pulse over that of the input pulse.

A basic shunt zener regulating circuit is shown in figure $5 \mathrm{a}$, with the a-c equivalent circuit of the zener junction shown in figure $5 \mathrm{~b}$. The junction capacitance, $C_{z}$, is of the order of a few hundred picofarads which results in the storage of charge in the junction.

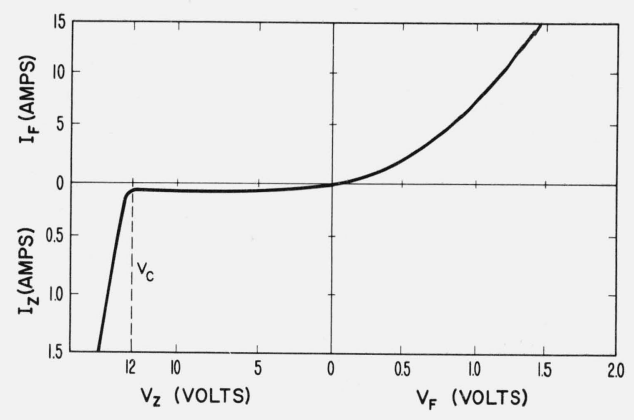

FIGURE 4. V-I Characteristics of zener diode.

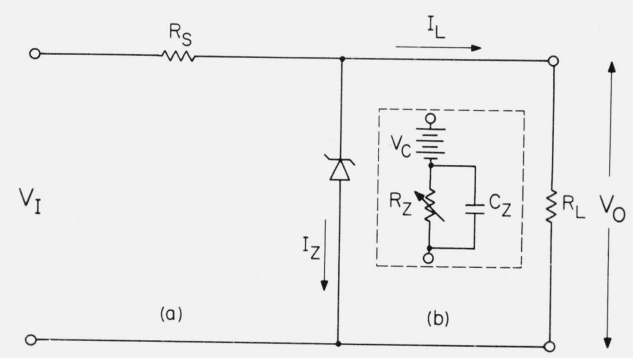

FIGURE 5 (a) Basic zener regulating circuit; (b) $A-C$ equivalent circuit of zener junction. 
This effect tends to limit the high frequency (and rise time) response. In designing the circuit for use as a d-c calibrated pulse voltage limiter, standard equations from handbooks were employed. The basic equation relating changes in output voltage, $V_{0}$, to changes in input voltage, $V_{i}$, load resistance, $R_{L}$, and temperature, $T$, is given by

$$
d V_{0}=\left(\frac{\delta V_{0}}{\delta V_{i}}\right) d V_{i}+\left(\frac{\delta V_{0}}{\delta R_{L}}\right) d R_{L}+\left(\frac{\delta V_{0}}{\delta T}\right) d T .
$$

In our application the second term in the above equation is not important because $R_{L}$ is constant and $d R_{L}=0$. The temperature effect on $V_{0}$ is, however, very important and required the use of diodes having low temperature coefficients. The importance of temperature coefficients is due to the fact that when calibrating $V_{0}$ using d-c voltage, the power dissipated in the junction may be several orders of magnitude greater than when the circuit is subsequently used with pulses. Temperature coefficients for the 10 and $50 \mathrm{~W}$ diodes used here are less than 0.1 percent $/{ }^{\circ} \mathrm{C}$. It was desired to limit $\left(\frac{\delta V_{0}}{\delta T}\right) d T$ to 0.1 percent and hence the maximum temperature rise for a $50 \mathrm{~W}$ diode is $1{ }^{\circ} \mathrm{C}$ which corresponds to $1 \mathrm{~W}$ power dissipation in the junction. (The thermal resistance of these diodes is $1{ }^{\circ} \mathrm{C} / \mathrm{W}$.)

During the d-c calibration of $V_{0}$, the magnitude of $V_{i}$ was adjusted so that the zener current $I_{z}$ was large enough to insure good regulation (low zener impedance) and at the same time not exceed $1 \mathrm{~W}$ of power in the junction. In subsequently using the calibrated circuit on a pulse generator, it was assumed that $V_{i \text { (pulse) }}$ could be set no closer than \pm 10 percent of $V_{i(d-c) \text {. It }}$ was required that the change in $V_{0}$ due to the uncertainty in $V_{i \text { (pulse) }}$ be limited to 0.1 percent. Thus the value of $\frac{\delta V_{0}}{\delta V_{i}}$ in eq (5) could be no greater than 0.01 Using this information it was possible to calculate the value of the series resistor, $R_{s}$, for each diode by use of the equation

$$
\frac{\delta V_{0}}{\delta V_{i}}=\frac{1}{1+R_{s}\left(\frac{1}{R_{Z}}+\frac{1}{R_{L}}\right)} .
$$

In a typical example for a $12 \mathrm{~V}, 10 \mathrm{~W}$ zener and a $50 \Omega$ load, the value of $R_{s}$ is $400 \Omega$. The voltage drop across $R_{s}$ is approximately $120 \mathrm{~V}$ and it must absorb $25 \mathrm{~W}$ of power. The same degree of regulation can be achieved with less power dissipation and voltage drop in $R_{s}$ by connecting a second higher voltage zener circuit ahead of the first as shown in figure 6 . The regulation required for each circuit is now only 0.1 (10 percent), and the values of $R_{s_{1}}$ and $R_{s_{2}}$ can be reduced to approximately $40 \Omega$ each. The rise time of the double diode circuit is $50 \mathrm{nsec}$ as compared to $30 \mathrm{nsec}$ for the single diode.

Zener diodes are manufactured with breakdown voltages ranging from a few volts to well over $100 \mathrm{~V}$.

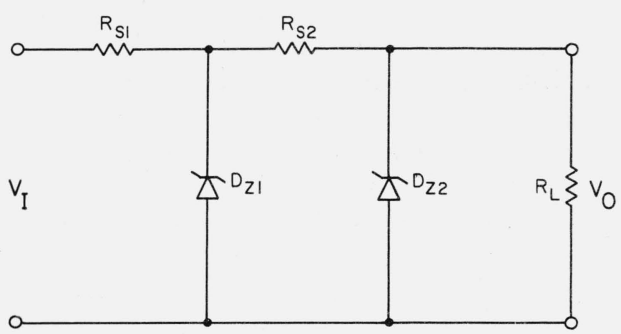

Figure 6. Double zener circuit.

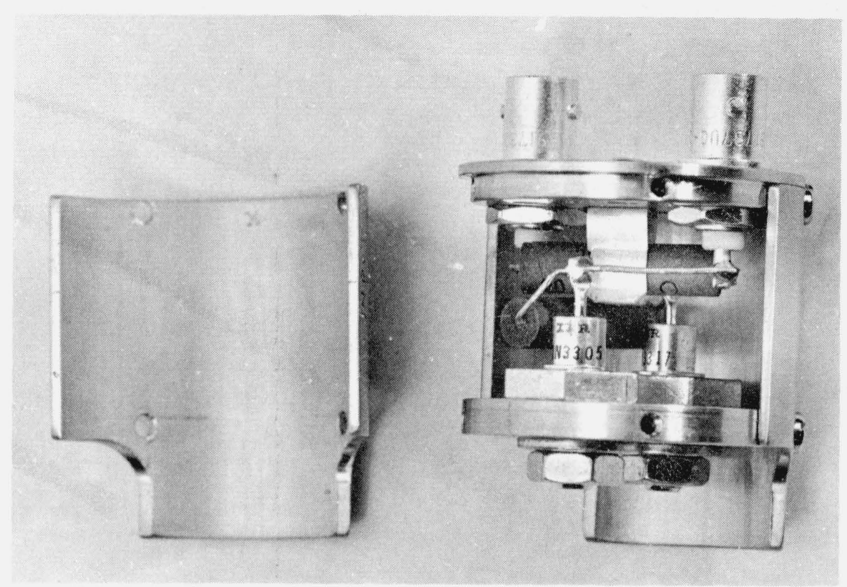

FIgURE 7. Mount for zener diodes and resistors used in the standard generating system.

Several circuits for various voltages in this range have been built and evaluated. In general, at the higher voltage levels it was found that the rise time could be improved by stacking several low voltage diodes in series rather than using a single high voltage unit.

Each zener circuit is mounted in a 2 -in. diam by 2 -in. high brass case as shown in figure 7 . The performance of the circuits was improved by immersing the circuit and case in liquid nitrogen. Nitrogen is allowed to fill the case through holes in the top and bottom. This served to insure that the 0.1 percent temperature effect was not exceeded and allowed the series resistor, $R_{s}$, to be smaller in physical size.

\section{Performance Characteristics and Results of Intercomparison}

As mentioned earlier the estimated uncertainty of the standard measuring system using the 1N270 diode is no greater than 0.05 percent $\pm 20 \mathrm{mV}$. Due to the fixed $20 \mathrm{mV}$ uncertainty and a maximum allowable error of 0.5 percent, the lower limit on the useful voltage range is $5 \mathrm{~V}$. The upper limit of $100 \mathrm{~V}$ is fixed by the maximum allowed reverse voltage of the diode. The rise time of the circuit is $10 \mathrm{nsec}$ and the minimum width of the peak of the pulse is 10 nsec. 
Tests were made on the standard measurement system to determine the effect of pulse duty cycle on the accuracy. Within the limits of precision, the measuring system was found to be insensitive to duty cycle in the range $10^{-5}$ to $5 \times 10^{-1}$. These tests were made by varying separately both the pulse duration and the repetition rate.

In the zener circuits, as in the measuring system, the effects of varying the duty cycle were negligible. The errors in the system include the 0.1 percent due to temperature effects, the 0.1 percent due to the uncertainty in setting $V_{i \text { (pulse) }}$ equal to $V_{i(\mathrm{~d} \text {-c) }}$ and the 0.05 percent error in the d-c voltmeter used to measure the limiting voltage, $V_{0}$. Thus, the overall estimated accuracy is 0.25 percent.

The zener circuits may also be used to simply regulate the output of a pulse generator. In this application variations in the output amplitude of the pulse generator are reduced by a factor of 100 to 1 at the output of the zener circuit. Higher regulation can be obtained by increasing the value of the source resistor, $R_{s}$.

Comparison measurements have been made between the two systems and with the NBS a-c and CW rf voltage standards. At $100 \mathrm{~Hz}$ the disagreement was less than 0.1 percent, at $10 \mathrm{MHz}$ and $30 \mathrm{MHz}$ less than 0.3 percent, and at $100 \mathrm{MHz}$ less than 2.5 percent. The uncertainty of the $100 \mathrm{MHz}$ rf CW thermal voltmeter used in the comparison was approximately 1 percent.

Agreement between the standard measurement system and the standard generating system was within 0.3 percent at all voltage levels where comparison was made. These voltage levels were $6 \mathrm{~V}, 12 \mathrm{~V}, 24 \mathrm{~V}$, $47 \mathrm{~V}$, and $100 \mathrm{~V}$.

A photograph of the console containing both standard systems is shown in figure 8 .

(Paper 70Cl-167)

\section{Conclusion}

The accuracies of the two systems for measurement and standardization of peak pulse voltage have been reasonably well established to be within 0.25 percent. These systems are considered an adequate basis for

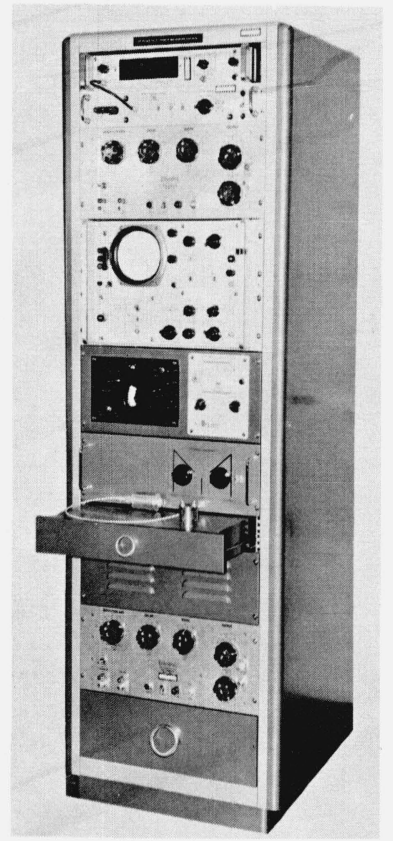

FIGURE 8. Accessory equipment for both pulse voltage standards. Included are the digital voltmeter, pulse generator, oscilloscope, and adjustable d-c bias supply.

establishment of a calibration service for presently available commercial instruments.

Further work is planned to improve the performance characteristics of the systems and to develop new superior systems. Extension of the voltage range above the present $100 \mathrm{~V}$ will be an important part of this work.

\section{References}

[1] Standards on pulses: definition of terms, Proc. IRE, 39, No. 6, (June 1951).

[2] F. E. Terman and J. M. Pettit, Electronic Measurements, pp. 34-36, (McGraw.Hill Book Company, 2d ed., 1952).

[3] M. C. Selby, High frequency voltage measurement, NBS Circular 481, (Sept. 1, 1949).

[4] Charles L. Alley and Kenneth W. Atwood, Electronic Engineering, p. 64, (John Wiley \& Sons, Inc., New York, N.Y., 1962).

[5] Aldert Van Der Ziel, Solid State Physical Electronics, p. 287, (Prentice-Hall, Inc., 1957). 